\title{
Systemic COVID-19 Vaccination also Enhances the Humoral Immune Response after SARS-CoV-2 Infection: A Population Study from a Hospital in Poland. Criteria for COVID-19 Reimmunization Are Needed
}

Piotr Kosiorek ( $\nabla$ pkosiorek@onkologia.bialystok.pl )

Oncology Center Bialystok

Dorota Elżbieta Kazberuk

Oncology Center Bialystok

Anna Hryniewicz

Medical University of Białystok

Robert Milewski

Medical University of Białystok

Samuel Stróż

Medical University of Białystok

Anna Stasiak-Barmuta

Medical University of Białystok

\section{Research Article}

Keywords: COVID-19, BNT162b2, vaccination, SARS CoV-2, reimmunisation

Posted Date: February 17th, 2022

DOI: https://doi.org/10.21203/rs.3.rs-858160/v6

License: (c) (i) This work is licensed under a Creative Commons Attribution 4.0 International License.

Read Full License

Version of Record: A version of this preprint was published at Vaccines on February 19th, 2022. See the published version at https://doi.org/10.3390/vaccines10020334. 


\section{Systemic COVID-19 Vaccination also Enhances the Humoral Immune Response after}

\section{SARS-CoV-2 Infection: A Population Study from a Hospital in Poland. Criteria for}

\section{COVID-19 Reimmunization Are Needed}

Piotr Kosiorek ${ }^{1,5, *}$, Dorota Elżbieta Kazberuk ${ }^{2}$, Anna Hryniewicz ${ }^{3}$, Robert Milewski ${ }^{4}$, Samuel Stróż ${ }^{5}$ and Anna Stasiak-Barmuta ${ }^{5}$

1 Department of Emergency, Maria Sklodowska-Curie Bialystok Oncology Centre, Ogrodowa 12, 15-027, Białystok, Poland

2 Department of Radiotherapy, Maria Sklodowska-Curie Bialystok Oncology Centre, Ogrodowa 12, 15-027, Białystok, Poland

3 Department of Rehabilitation, Medical University of Białystok, Jana Kilińskiego 1, 15089, Białystok, Poland

4 Department of Statistics and Medical Informatics, Medical University of Białystok, Jana Kilińskiego 1, 15-089, Białystok, Poland

5 Department of Clinical Immunology, Medical University of Białystok, Jana Kilińskiego 1, 15-089, Białystok, Poland

* Correspondence: piotr.kosiorek@umb.edu.pl

\section{Abstract:}

Systemic vaccination with the BNT162b2 mRNA vaccine stimulates the humoral response. Our study aimed to compare the intensity of the humoral immune response, measured by SARS-CoV-2 IgG, SARS-CoV-2 IgM, and S-RBD neutralizing IgG antibody levels, after COVID-19 vaccination versus after SARS-CoV-2 infection. We analyzed 1060 people in the following groups: convalescents, healthy vaccinated individuals, individuals vaccinated with Comirnaty, AstraZeneca, Moderna, or Johnson \& Johnson, and vaccinated SARS-CoV-2 convalescents. The concentrations of SARS-CoV-2 IgG, SARS-CoV-2 IgM, and S-RBD neutralizing antibodies were estimated in the oncology hospital laboratory by chemiluminescent immunoassay (CLIA; MAGLUMI). Results: (1) We observed a rise in antibody response in both SARS-CoV-2 convalescent and COVID-19-vaccinated groups. (2) The levels of all antibody concentrations in vaccinated COVID-19 convalescents were significantly higher. (3) We differentiated asymptomatic SARS-CoV-2 convalescents from the 
control group. Our analysis suggests that monitoring SARS-CoV-2 IgG antibody concentrations is essential as an indicator of asymptomatic COVID-19 and as a measure of the effectiveness of the humoral response in convalescents and vaccinated people. Considering the time-limited effects of post-SARS-CoV-2 infection recovery or vaccination and the physiological half-life, among other factors, we suggest monitoring the IgG antibody level as a criterion for the next vaccination.

\section{Keywords: COVID-19, BNT162b2, vaccination, SARS CoV-2, reimmunisation}

\section{Introduction}

Vaccination is the best means of preventing infectious diseases. Currently, the PfizerBioNTech vaccine BNT162b2 (Comirnaty) [1] is recognized as among the most effective vaccines in preventing SARS-CoV-2 infection and severe COVID-19 [2-4]. Rising levels of specific antibodies- $\operatorname{IgM}$ and $\operatorname{IgG}$ - and S-RBD neutralizing IgG as a humoral immune response are effects of vaccination. A similar effect is observed after SARS-CoV-2 exposure (coronavirus disease 2019, COVID-19) [5]. It has been proved that in symptomatic SARSCoV-2 infections, the level of S-RBD neutralizing antibodies correlates with the severity of COVID-19 and with hospitalization [5,6]. This correlation was not observed either in asymptomatic convalescent COVID-19 patients or in healthy vaccinated individuals after the first dose of a double systemic vaccination scheme [7,8]. Previous research provided evidence that after a second systemic dose of the Pfizer-BioNTech mRNA vaccine, the neutralizing antibody level was lower than that in vaccinated convalescent COVID-19 patients [8]. Higher levels of neutralizing antibodies after SARS-CoV-2 exposure and COVID-19 vaccination have been detected in individuals with autoimmune disease $[9,10]$. Seroconversion to neutralizing antibodies is gradual and depends on the patient's clinical state resulting from immune defense mechanisms, such as neutralization, complement activation, and cell cytotoxicity (antibodydependent cellular cytotoxicity, ADCC) [7,11,12]. In rare situations, SARS-CoV-2 infection 
gives rise to systemic inflammatory response syndrome (SIRS) with systemic inflammatory multiorgan dysfunction (MODS) [13]. Depending on the phase and intensity of the inflammatory response, administering convalescent serum plasma antibodies as a COVID-19 treatment may reduce or strengthen the inflammation [14]. There is much controversy involving the use of convalescent-derived serum plasma for COVID-19 therapy [14]. Serum plasma antibodies are very effective at an early stage of COVID-19, when the inflammation process has not yet involved elements of the humoral response [12,14,15].

The evaluation of systemic vaccinations against SARS-CoV-2 invariably raises questions about seroprotective antibody concentration levels and their half-life [11]. Aging of the immune system is, on the one hand, a risk factor for contracting COVID-19 and, on the other hand, determines poor postvaccination response [16]. The question then arises as to whether elderly individuals require an additional dose of the vaccine [16,17]. Should the same question be asked about immunosuppressed patients, people with immunodeficiency syndromes, post-transplantation patients, or oncologic or dialysis patients? Many published papers prove the safety of BNT162b2 vaccination in patients on dialysis [18] and after lymphoma therapy [19] and the safety of the third dose in kidney transplant recipients [20]. In healthy individuals, partial seroprotection, with a mean of approximately $53 \%$ (32-68\%, confidence interval 95\%), is reached 14 days after the first dose of the BNT162b2 vaccine. Seven days after the second dose, seroprotection reaches $95 \%$ [16]. Seroprotection is even higher for SARS-CoV-2 variants B.1.1.7 and B.1.351, with values of $75 \%$ and $97 \%$ after the first and second doses, respectively [21].

\section{Materials and Methods}

Our study observed a group of 1063 people, 786 females (74\%) and 277 males (26\%), in the age bracket between 18 and 89 years old. People over 50 years old constituted $45 \%(\mathrm{~N}=479)$ of the group. Because of different postvaccination reactions, three subjects were considered 
early hyper-responders based on their $\operatorname{IgM}$ and $\operatorname{IgG}$ levels, and we excluded them from further evaluation. The final group of 1060 subjects (Figure 1, Table 1) was divided into five subgroups (G0-G5): The control group G0 $(\mathrm{N}=154)$ was unvaccinated persons who showed no clinical signs of COVID-19 and tested negative for SARS-CoV-2 by RT-PCR. Group G1 $(\mathrm{N}=76)$ included fully symptomatic COVID-19 patients who tested positive for SARS-CoV2 by RT-PCR. Group G2 included 472 healthy individuals vaccinated with both doses of Comirnaty. Group G3 included 42 persons vaccinated with AstraZeneca $(\mathrm{N}=21)$, Moderna $(\mathrm{N}=19)$, or Johnson \& Johnson $(\mathrm{N}=2)$ according to the vaccination scheme. Group G4 included 312 COVID-19 convalescents vaccinated with Comirnaty. Group G5 consisted of 4 COVID-19 convalescents infected with SARS-CoV-2 after a complete systemic vaccination cycle. Two positive RT-PCR tests confirmed the infection.

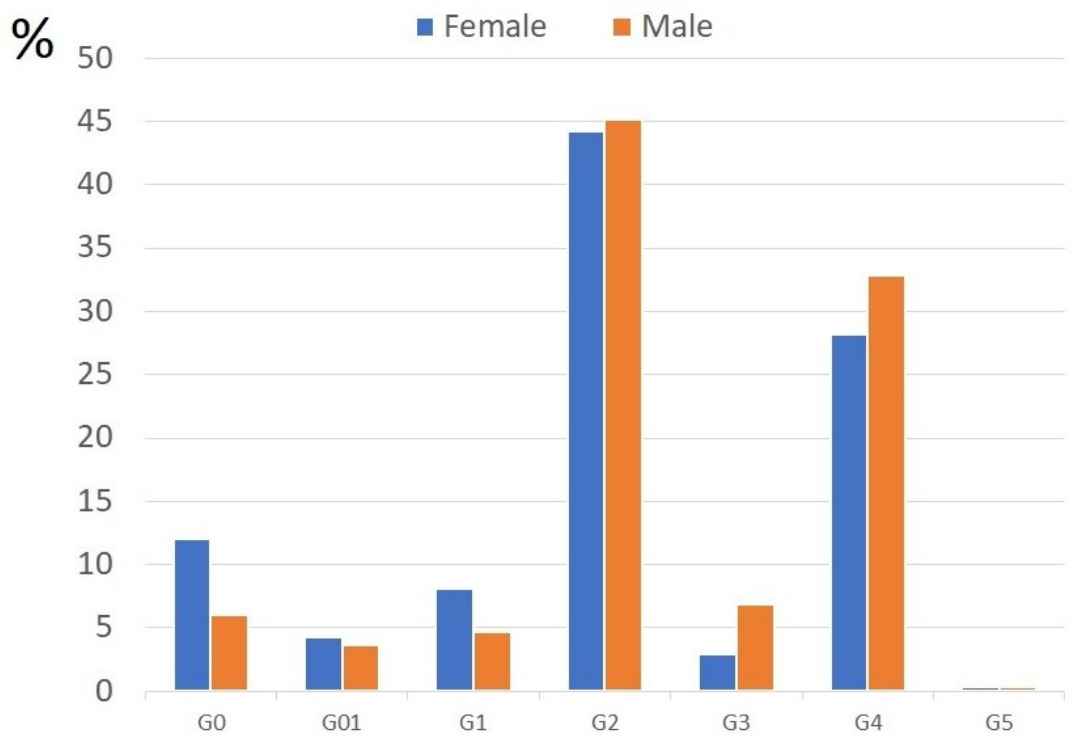

\section{Figure 1.}

Gender distribution in the study groups. There is no statistically significant difference among the study groups in terms of gender. 
Table 1. Population characteristics and outcomes in the case-control study.

\begin{tabular}{|c|c|c|c|c|c|c|c|c|}
\hline Groups & Participants & G0 & G01 & G1 & G2 & G3 & G4 & G5 \\
\hline All & 1060 & 111 & 43 & 76 & 472 & 42 & 312 & 4 \\
\hline$\%$ & 100 & 10.69 & 4.05 & 7.17 & 44.52 & 3.96 & 29.43 & 0.38 \\
\hline 2020 & $499(47.07 \%)$ & 51 & 14 & 30 & 215 & 7 & 179 & 3 \\
\hline 2021 & $561(52.92 \%)$ & 60 & 29 & 46 & 257 & 35 & 133 & 1 \\
\hline \multicolumn{9}{|l|}{ Sex } \\
\hline Female & $783(73.87 \%)$ & 94 & 10 & 33 & 63 & 346 & 23 & 3 \\
\hline Vaccinated & $594(75.86 \%)$ & 0 & 0 & 0 & 347 & 23 & 221 & 3 \\
\hline Unvaccinated & $189(24.14 \%)$ & 93 & 33 & 63 & 0 & 0 & 0 & 0 \\
\hline Male & $277(26.13 \%)$ & 18 & 33 & 10 & 125 & 19 & 91 & 1 \\
\hline Vaccinated & $236(85.19 \%)$ & 0 & 0 & 0 & 125 & 19 & 91 & 1 \\
\hline Unvaccinated & $41(14.8 \%)$ & 18 & 10 & 13 & 0 & 0 & 0 & 0 \\
\hline \multicolumn{9}{|l|}{ Age range } \\
\hline$<35$ & $220(20.75 \%)$ & 39 & 8 & 20 & 102 & 4 & 47 & 0 \\
\hline $36-49$ & $361(34.06 \%)$ & 39 & 12 & 23 & 163 & 15 & 109 & 0 \\
\hline$>50$ & $479(45.18 \%)$ & 34 & 23 & 33 & 206 & 23 & 156 & 4 \\
\hline \multicolumn{9}{|c|}{ COVID-19 test } \\
\hline Negative & $671(63.3 \%)$ & 111 & 43 & 0 & 471 & 35 & 11 & 0 \\
\hline Positive & $389(36.69 \%)$ & 0 & 0 & 76 & 1 & 7 & 301 & 4 \\
\hline \multicolumn{9}{|c|}{ COVID-19 response } \\
\hline No & $662(62.45 \%)$ & 111 & 43 & 0 & 470 & 35 & 2 & 0 \\
\hline $30-50$ days & $18(1.69 \%)$ & 0 & 0 & 13 & 1 & 2 & 1 & 2 \\
\hline 60-90 days & $81(7.64 \%)$ & 0 & 0 & 18 & 0 & 3 & 58 & 2 \\
\hline$>180$ days & $299(28.2 \%)$ & 0 & 0 & 45 & 1 & 0 & 251 & 0 \\
\hline \multicolumn{9}{|c|}{ Vaccinated } \\
\hline No & $233(21.79 \%)$ & 111 & 43 & 76 & 0 & 0 & 0 & 0 \\
\hline Yes & $827(78.01 \%)$ & 0 & 0 & 0 & 472 & 24 & 312 & 4 \\
\hline Comirnaty & $787(74.2 \%)$ & 0 & 0 & 0 & 472 & 0 & 312 & 4 \\
\hline AstraZeneca & $21(1.98 \%)$ & 0 & 0 & 0 & 0 & 21 & 0 & 0 \\
\hline Moderna & $19(1.79 \%)$ & 0 & 0 & 0 & 0 & 19 & 0 & 0 \\
\hline $\mathrm{J} \& \mathrm{~J}$ & $2(0.19 \%)$ & 0 & 0 & 0 & 0 & 2 & 0 & 0 \\
\hline \multicolumn{9}{|c|}{ Vaccinated response } \\
\hline No & $749(70.66 \%)$ & 111 & 43 & 76 & 288 & 31 & 197 & 2 \\
\hline$<14$ days & $58(5.47 \%)$ & 0 & 0 & 0 & 28 & 4 & 26 & 0 \\
\hline 14-90 days & $183(17.3 \%)$ & 0 & 0 & 0 & 111 & 0 & 64 & 1 \\
\hline
\end{tabular}




\begin{tabular}{|c|c|c|c|c|c|c|c|c|}
\hline$>90$ days & $70(6.6 \%)$ & 0 & 0 & 0 & 45 & 0 & 25 & 1 \\
\hline IgG (AU/mL) & 1060 & & & & & & & \\
\hline$<1.0$ & $685(64.62 \%)$ & 111 & 19 & 41 & 420 & 27 & 192 & 3 \\
\hline$>0.2$ & $375(35.37 \%)$ & 0 & 43 & 42 & 103 & 19 & 167 & 1 \\
\hline$>1.0$ & $247(23.30 \%)$ & 0 & 24 & 35 & 52 & 15 & 120 & 1 \\
\hline IgM (AU/mL) & 1060 & & & & & & & \\
\hline$<1.0$ & $879(82.92 \%)$ & 103 & 33 & 65 & 397 & 36 & 242 & 3 \\
\hline$>1.0$ & $181(17.07 \%)$ & 18 & & 11 & 75 & 6 & 70 & 1 \\
\hline S-RBD IgG (AU/mL) & 546 & & & & & & & \\
\hline$<1.0$ & $81(7.64 \%)$ & 31 & 2 & 6 & 31 & 4 & 7 & 0 \\
\hline$>1.0$ & $465(43.86 \%)$ & 20 & 22 & 33 & 220 & 21 & 147 & 2 \\
\hline$>50$ & $295(27.83 \%)$ & 0 & 3 & 11 & 154 & 8 & 117 & 2 \\
\hline$>100$ & $246(23.20 \%)$ & 0 & 3 & 8 & 126 & 6 & 101 & 2 \\
\hline$>500$ & $57(5.38 \%)$ & 0 & 0 & 1 & 14 & 4 & 36 & 2 \\
\hline$>1000$ & $38(3.58 \%)$ & 0 & 0 & 0 & 10 & 3 & 23 & 2 \\
\hline
\end{tabular}

The average age of 1060 participants is 47.5 years. The population is normally distributed. Preferential extension of vaccinations to only some age groups in Poland prevented us from correctly interpreting these data. No relationship with gender was observed in the analyzed groups. All antibody concentration results are in AU/mL. The COVID-19 test indicates population groups testing positive or negative by RT-PCR. COVID-19 response indicates the population groups where antibodies had a temporal correlation with infection, as measured by a positive test. Vaccinated COVID-19 represents the vaccine population groups. Vaccination response refers to the population groups with a temporal correlation with the performance of the vaccination. We rejected 3 persons from the 1063 participants regarded as hyper-responders based on IgM and IgG because markedly higher antibody concentrations were measured $(>2000 \mathrm{AU} / \mathrm{mL}$ IgG and $>700$ and $>300 \mathrm{AU} / \mathrm{mL} \operatorname{IgM})$. The person with an over-response of IgG was also an early responder ( $<14$ days) due to an autoimmune disease. The G5 group included too few people to draw conclusions, but four people had high levels of S-RBD IgG and low levels of SARS-CoV-2 IgM and IgG. This phenomenon seems to be related to the loss of antibodies following a previous complete vaccination and an incidence of SARS-CoV-2 infection. This study is most relevant for Delta virus variants in Poland. We were unable to determine the type of virus variants in our laboratory.

After a retrospective analysis of concentrations of SARS-CoV-2 IgG antibodies in group G0 $(\mathrm{N}=154)$, we identified subgroup $\mathrm{G} 01$, which comprised 43 persons who had concentrations of specific IgG antibodies that were higher than the cut-off value $(0.2 \mathrm{AU} / \mathrm{mL})$. The existence of specific SARS-CoV-2 antibodies was regarded as evidence of viral contact, and the patients were classified as SARS-CoV-2 convalescents with no symptomatic COVID-19 
history. Finally, G0 = 111. Among 43 analyzed cases (G01), 13 persons were in the seroconversion phase (positive for both $\operatorname{IgM}>1.0 \mathrm{AU} / \mathrm{mL}$ and $\operatorname{IgG}>0.2 \mathrm{AU} / \mathrm{mL}$ ), 30 persons were in a late phase of producing secondary antibodies (positive for $\operatorname{IgG}$, negative for $\operatorname{IgM}$ ), and 8 persons were in an early phase of the humoral viral response (positive for IgM, negative for $\operatorname{IgG})$.

\subsection{Materials}

Our study materials were blood specimens taken through venipuncture sampling. The concentration of antibodies was evaluated $4 \mathrm{~h}$ after blood collection. If an immediate assessment was not possible, the serum was collected and stored at $-80{ }^{\circ} \mathrm{C}$.

\subsection{Methods}

All 1063 participants had elevated levels of $\operatorname{IgM}$ and IgG antibodies oriented specifically towards SARS-CoV-2; in 546 subjects, anti-S (S-RBD) IgG antibodies were detected by chemiluminescent immunoassay (CLIA; MAGLUMI, Snibe Diagnostic, Shenzhen China).

Results greater than or equal to $1.0 \mathrm{AU} / \mathrm{mL}$ SARS-CoV-2 IgG, IgM, and S-RBD were considered indicative of a reaction and regarded as positive according to the manufacturer's protocol.

Of the study participants, 827 were vaccinated, including 787 persons who received Comirnaty. BNT162b2 is $95 \%$ effective in preventing COVID-19, and similar vaccine efficacy is observed across subgroups defined by age, sex, race, and ethnicity [1].

The Bioethics Commission of Medical University approved our research.

\section{Statistical analysis}

Nonparametric statistical methods were applied for the case-control analyses because many of the considered variables were not normally distributed. The Mann-Whitney test was 
adopted to compare two groups to each other, whereas the Kruskal-Wallis test was applied to assess more than two groups. Results were considered statistically significant when $p<0.05$. We used PQStat GEN 4.4.12 program (PQStat Software, Poznan, Poland) and Tibco Statistics 13.3 for statistical analyses.

\section{Results}

After evaluating the concentrations of specific antibodies in the control group of healthy unvaccinated subjects, we identified a subgroup of SARS-CoV-2 convalescents (G01) after an asymptomatic case of COVID-19. The concentration of IgG antibodies in that subgroup was comparable to the concentration observed in COVID-19 convalescents with fully symptomatic disease confirmed by a positive RT-PCR test.
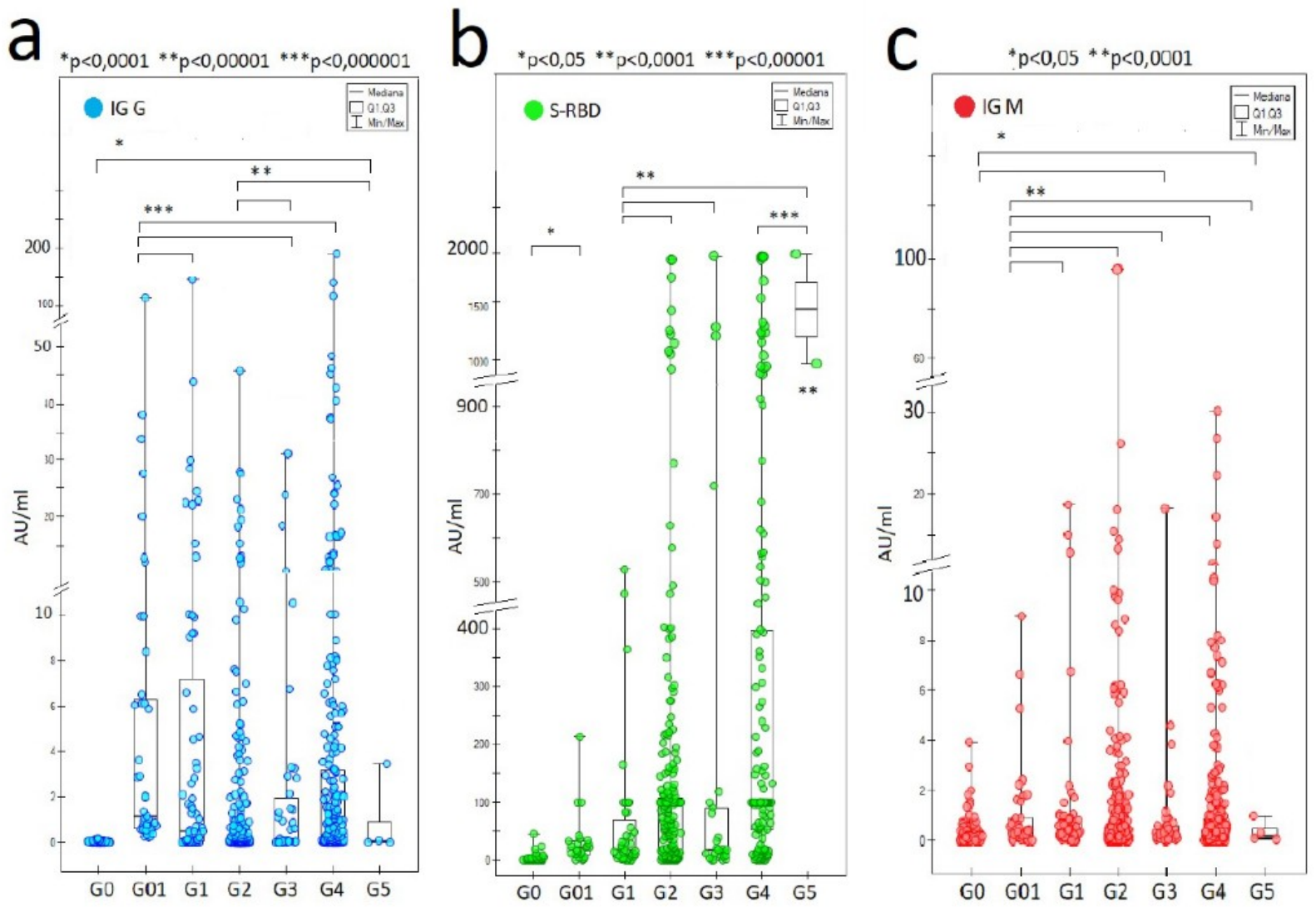

\section{Figure 2.}

The absolute concentrations of SARS-CoV-2 $\operatorname{IgG}(\mathbf{a}), \operatorname{IgM}(\mathbf{c})$, and S-RBD $\operatorname{IgG}(\mathbf{b})$ antibodies in each study group G0-G5 (* $p$, Kruskal-Wallis ANOVA). 
Our research showed the presence of SARS-CoV-2 antibodies in both the convalescent and vaccinated COVID-19 groups, and the concentration of antibodies decreased with time in both groups (Figure 2).

Significantly higher IgG- and IgM-specific antibody concentrations were detected in vaccinated COVID-19 convalescents (G4) (Figure 3).
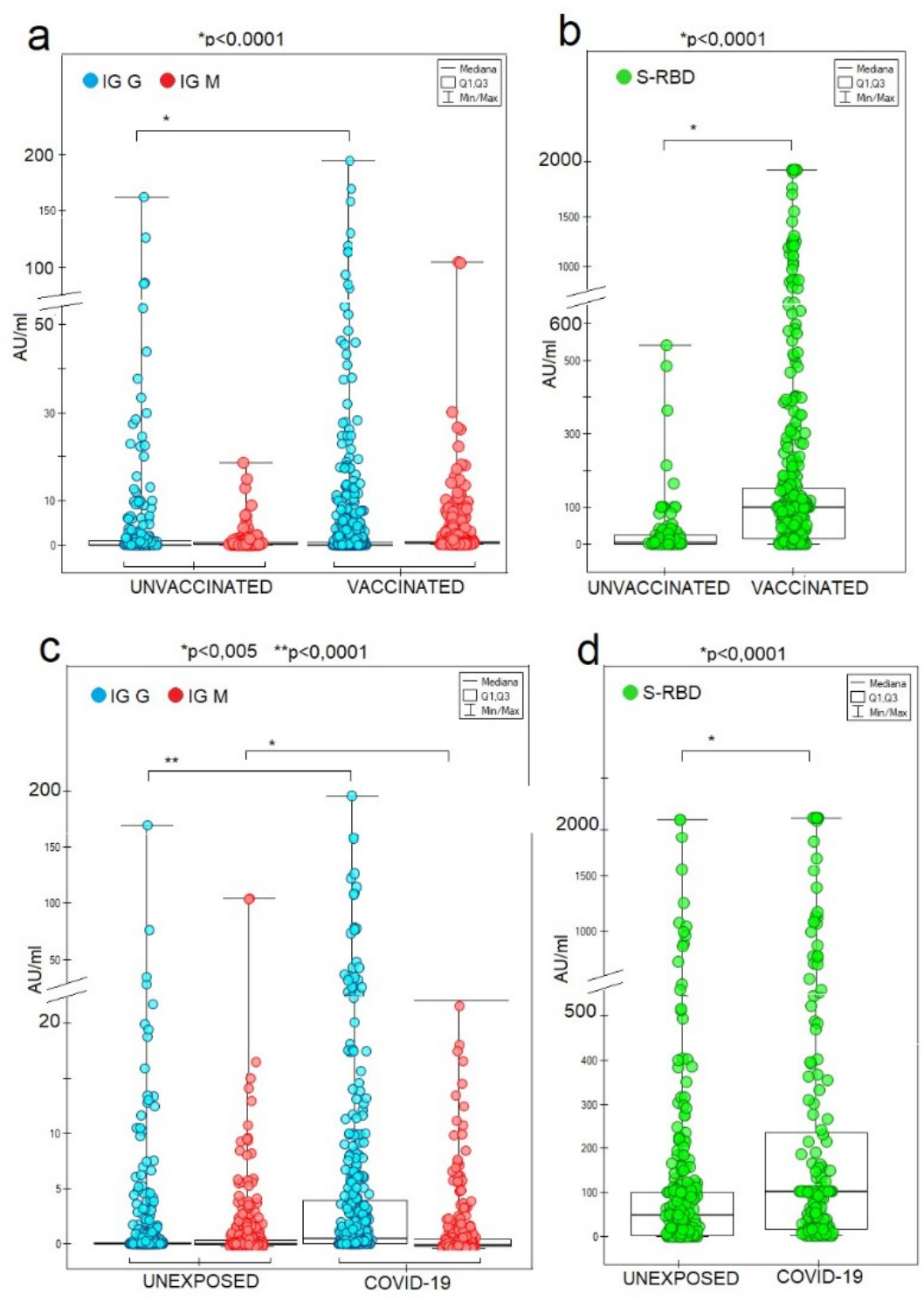


\section{Figure 3.}

The absolute concentrations of SARS-CoV-2 IgG and IgM antibodies (a,c) and S-RBD neutralizing IgG (b,d) for vaccinated and COVID-19 individuals ( $* p$, Kruskal-Wallis ANOVA). Unexposed COVID-19 group includes people who tested negative for COVID-19 and people with no history of being infected before measuring the antibodies.

After we analyzed the strength of specific IgG and IgM generation after vaccination, we did not find any significant difference between the studied vaccines (Pfizer, AstraZeneca, and Moderna). The sample size of the Johnson \& Johnson vaccine recipients $(\mathrm{N}=2)$ was too small to render a meaningful result; hence, we excluded these individuals from further analysis.

After we analyzed the strength of specific IgG and IgM generation after vaccination, we did not find any significant difference between the studied vaccines (Pfizer, AstraZeneca, Moderna). The sample size of the Johnson \& Johnson vaccine recipients $(\mathrm{N}=2)$ was too small to render a meaningful result; hence, we excluded it from further analysis.
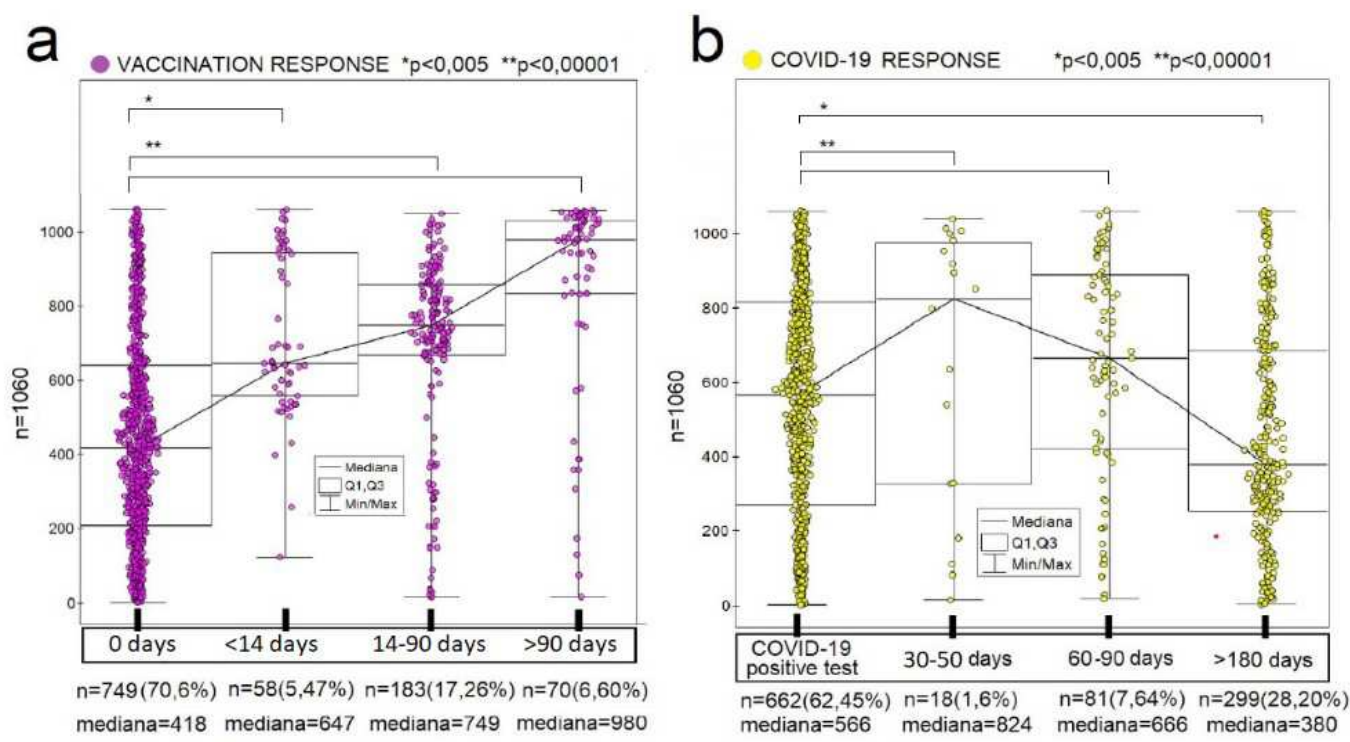

\section{Figure}

4.

The determination of antibodies in the study groups at intervals (a) after full vaccination and (b) after obtaining a positive result for SARS-CoV-2 (COVID-19) (* $p$, Kruskal-Wallis). 
Figure 4 shows the levels of antibodies in (a) individuals evaluated at time intervals after full vaccination and (b) after a positive RT-PCR test result for SARS-CoV-2 (COVID-19). The completed analysis showed a linear dependence over time for both vaccination response and natural response to viral infection after clinically mild or moderate COVID-19. We observed the effect mentioned above in each studied group for up to 180 days postexposure and up to 90 days postvaccination.

\section{Discussion}

The evaluation of concentrations of IgG and IgM antibodies specific to SARS-CoV-2 allowed us to identify a new subgroup among healthy unvaccinated controls: subjects who had a previous asymptomatic SARS-CoV-2 infection. Every patient in this subgroup presented lower concentrations of specific SARS-CoV-2 IgG antibodies than the cut-off established for a positive result in laboratory tests, which is between 0.2 and $1.0 \mathrm{AU} / \mathrm{mL}$.

Based on the concentration of neutralizing antibodies, we propose the following hierarchy of activation of the humoral response: unvaccinated $(\mathrm{G} 0)<$ convalescents $(\mathrm{G} 1)<$ vaccinated $(\mathrm{G} 2, \mathrm{G} 3)<$ vaccinated convalescents $(\mathrm{G} 4)<$ patients with SARS-CoV-2 infection after being fully vaccinated (G5).

Group G4 was exposed to the virus three times: the first time was when they were infected with the SARS-CoV-2 virus, and the following two times were when they were injected through immunization. Every contact with the antigen altered the immune system, causing a burst of antibody production lasting up to 14 days [22,23]. The triple exposure of this group caused the most significant rise in neutralizing antibodies among all groups observed.

Although group G5 $(\mathrm{N}=4)$, vaccinated people who developed fully symptomatic COVID-19, was excluded from further statistical analysis due to its small size, the results that we obtained 
for this group correlate with literature data, which shows the most significant rise in antibody concentration in vaccinated and infected people $[8,21]$.

\subsection{Who Needs the Third Dose of the Vaccine?}

The synthesis of IgM antibodies is the first phase of the humoral response after initial contact with the antigen. IgG antibody synthesis occurs after the second or repeated contact with the same antigen. The analysis of concentrations of specific IgG and IgM SARS-CoV-2 antibodies and their mutual dependence enabled the identification of early and late phases of the humoral response to SARS-CoV-2 infection and the COVID-19 vaccine [17,21]. Indirectly, it allowed us to identify individuals who had asymptomatic COVID-19. One should remember that the timing of blood sample collection is fundamental for the correct evaluation of the concentrations of $\operatorname{IgM}$ and $\operatorname{IgG}$ antibodies. This timing depends on the patient's clinical status and dynamic changes in the immune system, e.g., after SARS-CoV-2 infection, and can effectively optimize the planning of the next vaccine dose against the Delta variant [22]. Of course, such an evaluation needs to compare clinical examinations, identify infection symptoms or lack thereof, and perform a serological investigation for the presence or lack of virus antigens.

In Israel, we observed an increase in SARS infection among people vaccinated with the BNT1262b vaccine 146 days after vaccination [23]. This increase was the most pronounced in people over 60 [23]. In our opinion, it is reasonable to start measuring specific IgG (late effect) and IgM (early effect) antibodies for vaccination evaluation at the serological window of neutralizing antibody production. To date, data have shown that the humoral response of neutralizing antibodies after vaccination is rapid and decreases. In addition, in some people who have not been exposed to SARS-CoV-2, late humoral and cellular responses are detected, indicating widespread immunity to SARS in the population [24]. In summary, our results indicate the importance of evaluating and monitoring immunological response parameters in 
vaccinated healthy people and convalescents, which will help establish the need for third and subsequent doses of the vaccine against SARS-CoV-2.

\subsection{Conclusions}

Although our results could not be correlated with age, an increase in antibodies after systemic BNT162b2 vaccination, as indicated by other authors, confirms the benefits of monitoring antibody levels due to individual humoral responses and the age-associated decrease in immunity [25]. Studies by Lia et al. [26] indicate that specific SARS-CoV-2 IgG is a more sensitive measure than S-RBD antibodies for monitoring the humoral response in asymptomatic COVID-19 patients. The humoral response of cells after COVID-19 seems to be sufficient for over three months [27]. However, we noted that subjects in our hospital who had a mild illness were not wholly asymptomatic. We suggested individually checking antibodies strongly correlated with the patient's clinical condition. The research reported by Dan et al. [28] agrees with our observations that S-RBD antibodies are stable for up to 6 months. In conclusion, the determination of SARS-CoV-2 IgG provides more important information three months after suffering from the disease or receiving the vaccine. The manuscript was submitted as a preprint [29].

Author Contributions: P.K., A.H. and A.S.-B. analyzed the data and drafted the manuscript. R.M. participated in data analysis and extensively reviewed the manuscript. Other authors: D.E.K. and S.S. contributed to clinical and laboratory data acquisition and reviewed the manuscript. All authors have read and agreed to the published version of the manuscript.

Funding: The research did not receive specific funding but was performed as part of the work of the authors at Maria Sklodowska-Curie Bialystok Oncology Centre, Poland.

Institutional Review Board Statement: The Bioethics Commission of Medical University approved our research. Approval code: APK.002.267.2021; approval date: 29 April 2021. 
Informed Consent Statement: Written informed consent has been obtained from the patient(s) to publish this paper.

Data Availability Statement: Data are available on request.

Acknowledgments: We thank all health-care workers involved in examinations in Maria Sklodowska-Curie Bialystok Oncology Centre, Poland in Bialystok, Poland. All the individuals included in this work have consented to the acknowledgement.

Conflicts of Interest: The authors declare no conflict of interest.

\section{References}

1. Zhou, P. et al. A pneumonia outbreak associated with a new coronavirus of probable bat origin. Nature 579, 270-273, doi:10.1038/s41586-020-2012-7 (2020).

2. Arunachalam, P. S. et al. Systems vaccinology of the BNT162b2 mRNA vaccine in humans. Nature 596, 410-416, doi:10.1038/s41586-021-03791-x (2021).

3. Koch, T., Mellinghoff, S. C., Shamsrizi, P., Addo, M. M. \& Dahlke, C. Correlates of Vaccine-Induced Protection against SARS-CoV-2. Vaccines (Basel) 9, doi:10.3390/vaccines9030238 (2021).

4. Polack, F. P. et al. Safety and Efficacy of the BNT162b2 mRNA Covid-19 Vaccine. N Engl J Med 383, 2603-2615, doi:10.1056/NEJMoa2034577 (2020).

5. Long, Q. X. et al. Clinical and immunological assessment of asymptomatic SARS-CoV-2 infections. Nat Med 26, 1200-1204, doi:10.1038/s41591-020-0965$6(2020)$.

6. Lucas, C. et al. Delayed production of neutralizing antibodies correlates with fatal COVID-19. Nature Medicine 27, 1178-1186, doi:10.1038/s41591-021-01355-0 (2021).

7. Dufloo, J. et al. Asymptomatic and symptomatic SARS-CoV-2 infections elicit polyfunctional antibodies. Cell Rep Med 2, 100275, Doi: 10.1016/j.xcrm.2021.100275 (2021).

8. Vicenti, I. et al. The second dose of the BNT162b2 mRNA vaccine does not boost SARS-CoV-2 neutralizing antibody response in previously infected subjects. Infection, doi:10.1007/s15010-021-01680-z (2021).

9. Wang, E. Y. et al. Diverse functional autoantibodies in patients with COVID-19. Nature 595, 283-288, doi:10.1038/s41586-021-03631-y (2021).

10. Robbiani, D. F. et al. Convergent antibody responses to SARS-CoV-2 in convalescent individuals. Nature 584, 437-442, doi:10.1038/s41586-020-2456-9 (2020).

11. Becker, M. et al. Immune response to SARS-CoV-2 variants of concern in vaccinated individuals. Nature Communications 12, 3109, doi:10.1038/s41467021-23473-6 (2021). 
12. Tan, L. et al. Plasma therapy cured a COVID-19 patient with long duration of viral shedding for 49 days: The clinical features, laboratory tests, plasma therapy, and implications for public health management. MedComm (Beijing), doi:10.1002/mco2.2 (2020).

13. Jang, J. G., Hur, J., Hong, K. S., Lee, W. \& Ahn, J. H. Prognostic Accuracy of the SIRS, qSOFA, and NEWS for Early Detection of Clinical Deterioration in SARSCoV-2 Infected Patients. J Korean Med Sci 35, e234, doi:10.3346/jkms.2020.35. e234 (2020).

14. Arvin, A. M. et al. A perspective on potential antibody-dependent enhancement of SARS-CoV-2. Nature 584, 353-363, doi:10.1038/s41586-020-2538-8 (2020).

15. Kow, C. S. \& Hasan, S. S. Real-world effectiveness of BNT162b2 mRNA vaccine: a meta-analysis of large observational studies. Inflammopharmacology 29, 1075-1090, doi:10.1007/s10787-021-00839-2 (2021).

16. Kontopoulou, K. et al. Second dose of the BNT162b2 mRNA vaccine: Value of timely administration but questionable necessity among the seropositive. Vaccine 39, 5078-5081, Doi: 10.1016/j.vaccine.2021.07.065 (2021).

17. Polewska, K. et al. Safety and Tolerability of the BNT162b2 mRNA COVID-19 Vaccine in Dialyzed Patients. COViNEPH Project. Medicina (Kaunas) 57, doi:10.3390/medicina57070732 (2021).

18. Perry, C. et al. Efficacy of the BNT162b2 mRNA COVID-19 vaccine in patients with B-cell non-Hodgkin lymphoma. Blood Adv 5, 3053-3061, doi:10.1182/bloodadvances.2021005094 (2021).

19. Stumpf, J. et al. Cellular and Humoral Immune Responses after Three Doses of BNT162b2 mRNA SARS-Cov-2 Vaccine in Kidney Transplant. Transplantation, doi:10.1097/TP.0000000000003903 (2021).

20. Abu-Raddad, L. J. et al. Pfizer-BioNTech mRNA BNT162b2 Covid-19 vaccine protection against variants of concern after one versus two doses. J Travel Med 28, doi:10.1093/jtm/taab083 (2021).

21. Lou, B. et al. Serology characteristics of SARS-CoV-2 infection after exposure and post-symptom onset. Eur Respir J 56, doi:10.1183/13993003.00763-2020 (2020).

22. Gobbi, F. et al. Antibody response in individuals infected with SARS-CoV-2 early after the first dose of the BNT162b2 mRNA vaccine. J Infect, DOI: 10.1016/j.jinf.2021.08.008 (2021).

23. Israel, A. et al. Elapsed time since BNT162b2 vaccine and risk of SARS-CoV-2 infection in a large cohort. medRxiv, doi:10.1101/2021.08.03.21261496 (2021).

24. Grifoni, A. et al. SARS-CoV-2 human T cell epitopes: Adaptive immune response against COVID-19. Cell Host Microbe 29, 1076-1092, DOI: 10.1016/j.chom.2021.05.010 (2021).

25. The manuscript has been submitted as a preprint in the below link: https://www.researchsquare.com/article/rs-858160/v6. 


\section{Figures}

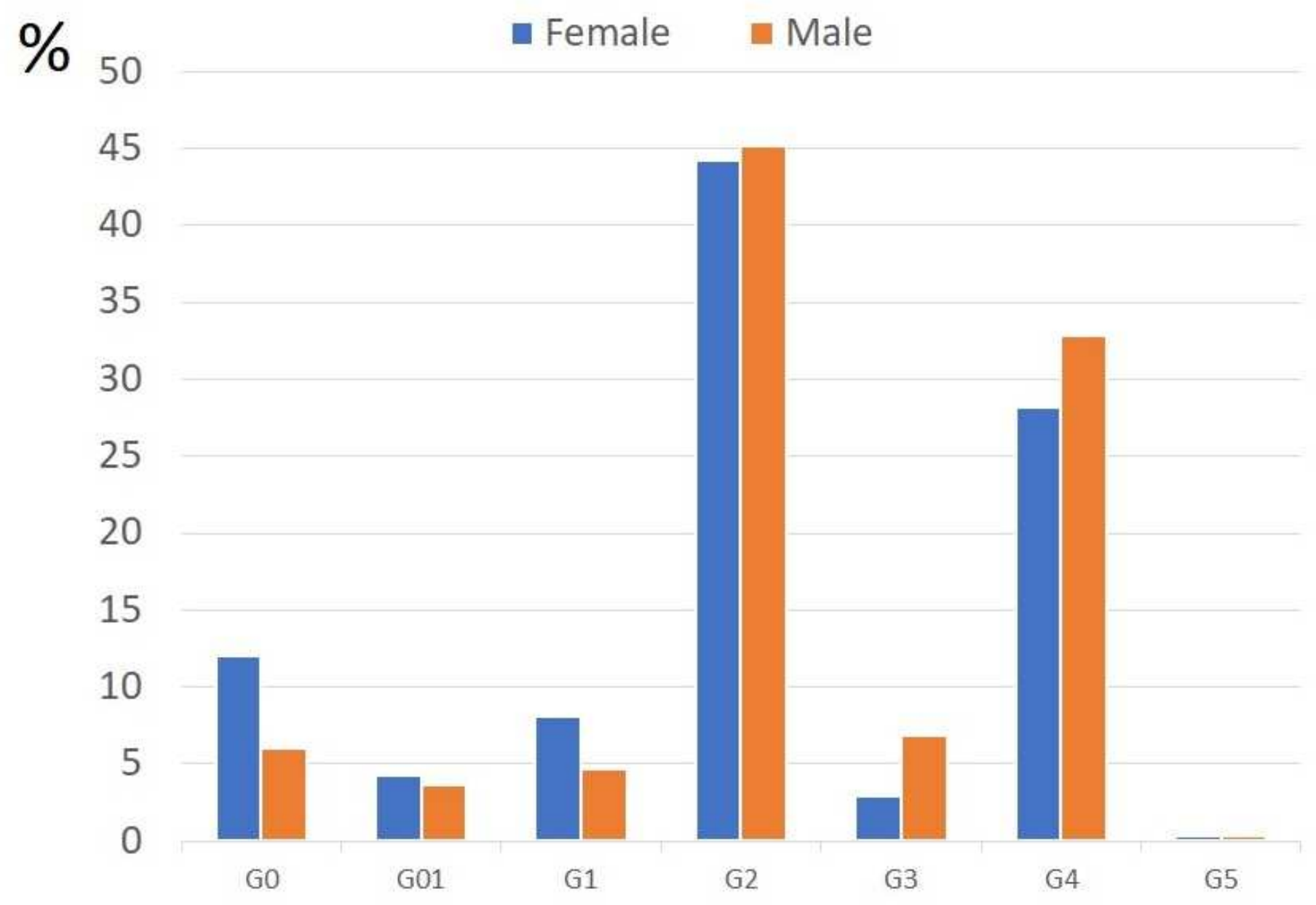

Figure 1

Gender distribution in the study groups. There is no statistically significant difference among the study groups in terms of gender. 

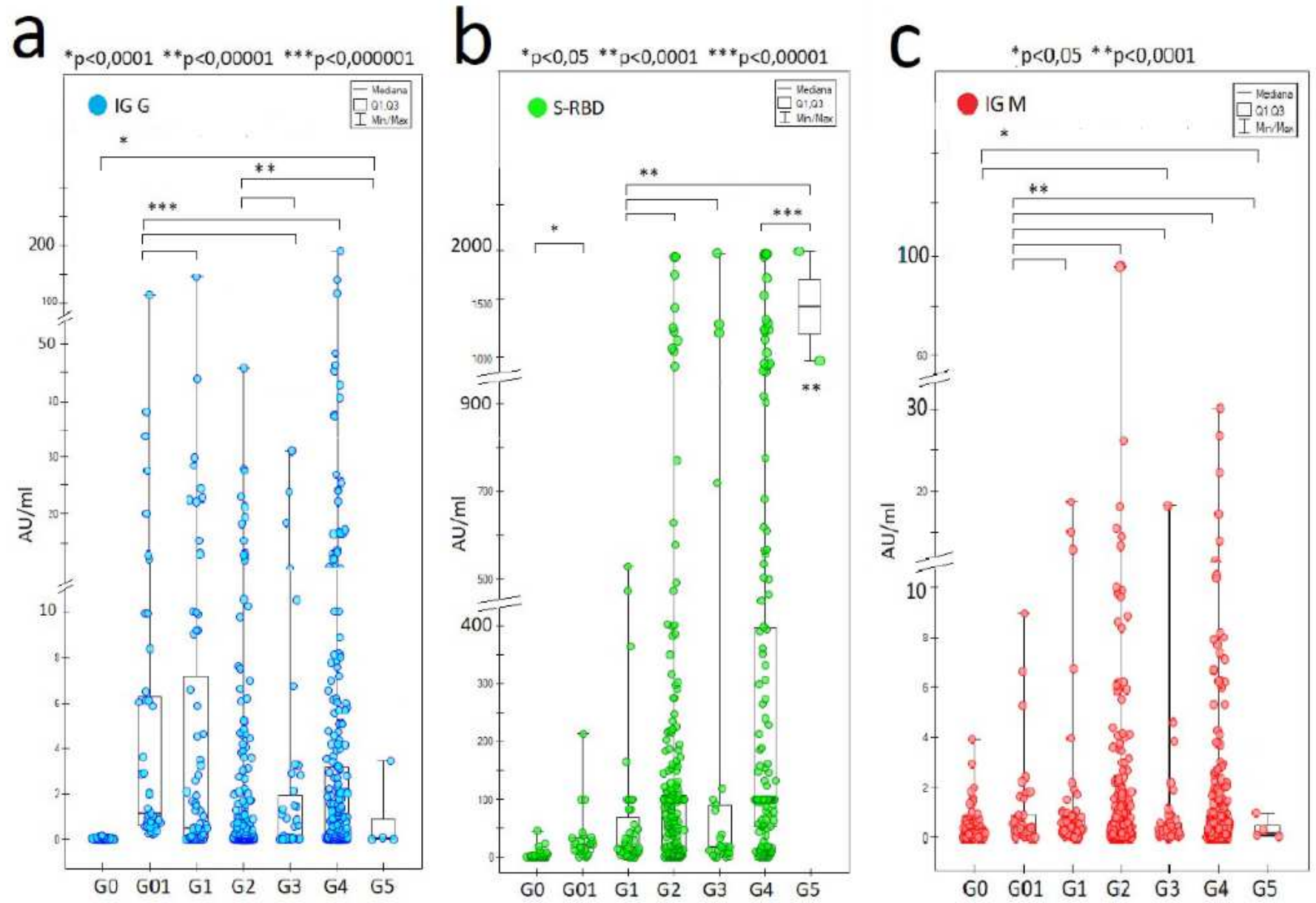

Figure 2

The absolute concentrations of SARS-CoV-2 IgG (a), IgM (c), and S-RBD IgG (b) antibodies in each study group G0-G5 (* $p$, Kruskal-Wallis ANOVA). 

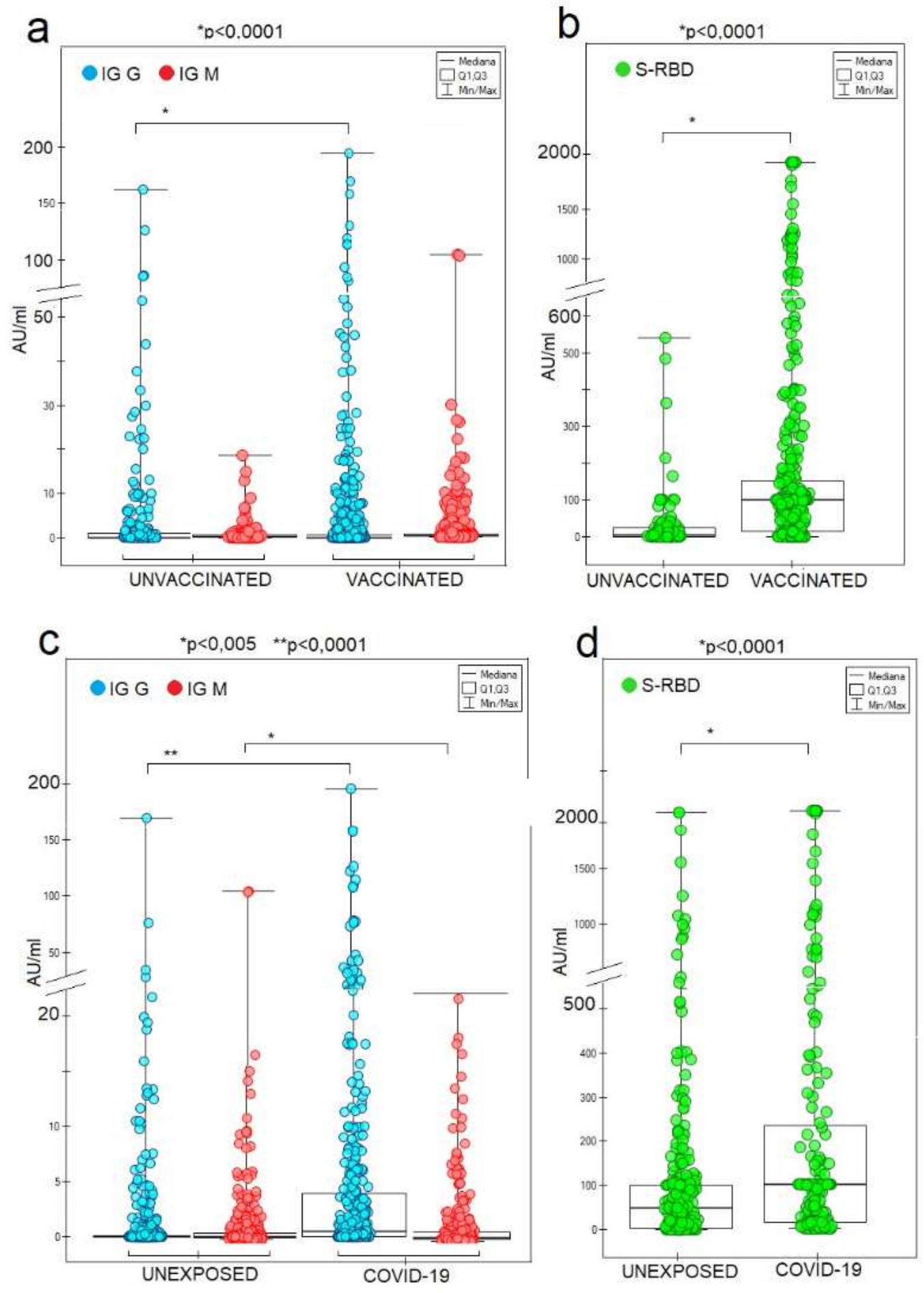

\section{Figure 3}

The absolute concentrations of SARS-CoV-2 IgG and IgM antibodies $(\mathbf{a}, \mathbf{c})$ and S-RBD neutralizing IgG (b,d) for vaccinated and COVID-19 individuals ( ${ }^{*} p$, Kruskal-Wallis ANOVA). Unexposed COVID-19 group includes people who tested negative for COVID-19 and people with no history of being infected before measuring the antibodies. 

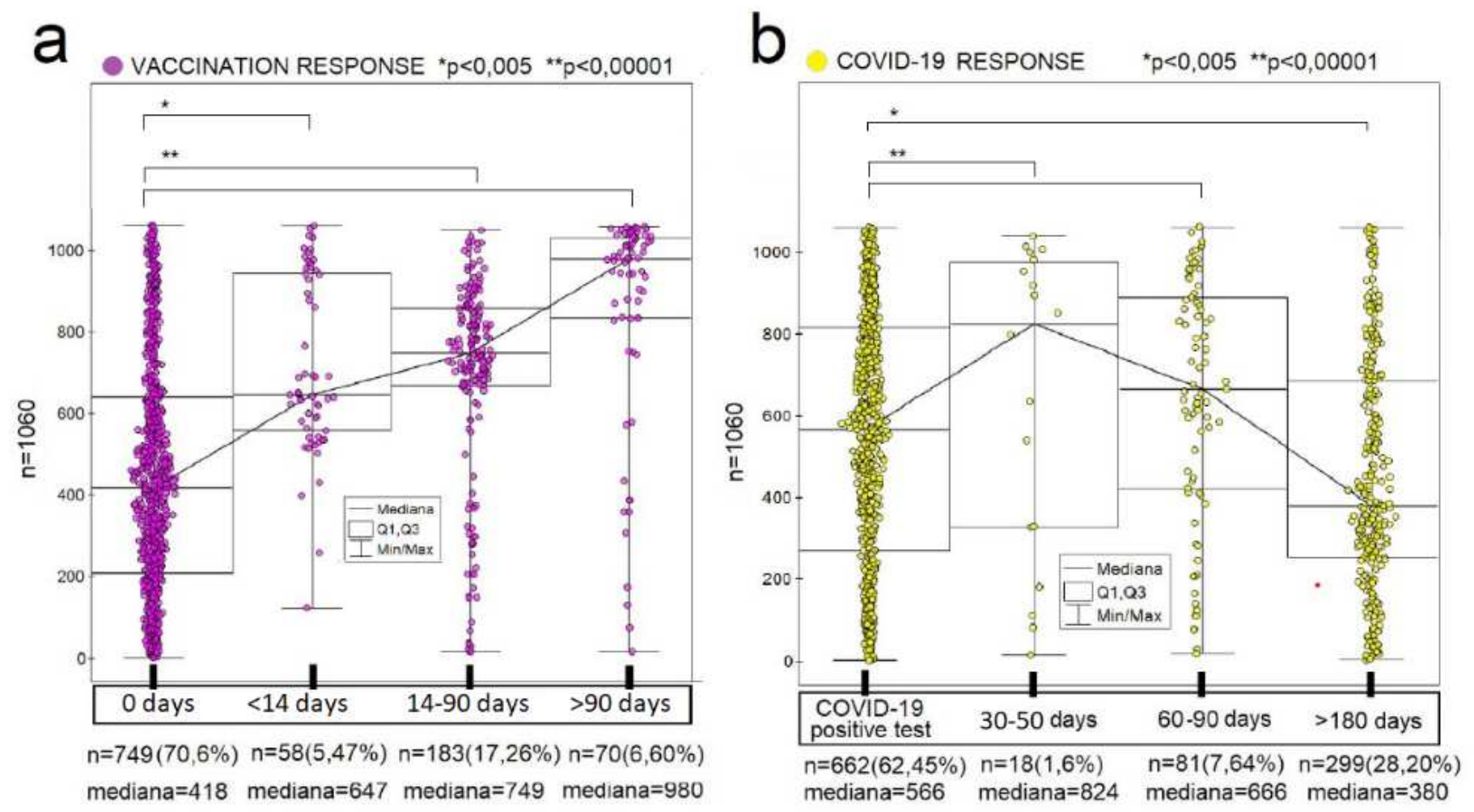

\section{Figure 4}

The determination of antibodies in the study groups at intervals (a) after full vaccination and (b) after obtaining a positive result for SARS-CoV-2 (COVID-19) ( ${ }^{*} p$, Kruskal-Wallis)

\section{Supplementary Files}

This is a list of supplementary files associated with this preprint. Click to download.

- graphicabstract1.jpg

- Table1x.odt 